\title{
Formación pre-grado en drogodependencias en las universidades españolas.
}

\author{
Daniel lloret Irles*; Mª Teresa Poveda Huertes**; Ma Carmen Soliveres Tomás** \\ Instituto de Investigación de Drogodependencias (INID). Universidad Miguel Hernández. \\ * Profesor. \\ ** Becario de investigación. \\ Enviar correspondencia a:
}

Daniel Lloret. Instituto de Investigación de Drogodependencias. Facultad de Medicina - Universidad Miguel Hernández. Ctra. de Valencia s/n. 03550 San Juan - Alicante.Tfno.- 9659193 19. Correo-e.- daniel.lloret@umh.es

Recibido: 1 de agosto de 2003. Aceptado: 6 de julio de 2004.

\section{RESUMEN}

Objetivo: El presente trabajo pretende analizar y describir la formación pre-grado en drogodependencias ofertada por las universidades españolas. Método: Los autores han revisado los planes de estudios de las 139 facultades y escuelas universitarias españolas que ofrecen las titulaciones de medicina, psicología, enfermería y/o trabajo social. Un total de 93 asignaturas monográficas sobre drogas impartidas por 58 facultades y escuelas universitarias han sido incluidas en este estudio. Resultados: Atendiendo al número de asignaturas, observamos que la titulación de enfermería es la que ofrece el mayor número. Las titulaciones de medicina y psicología se sitúan en segundo y tercer lugar, con 26 y 24 asignaturas respectivamente. Por último, encontramos trabajo social con 6 asignaturas impartidas. Siguiendo un criterio geográfico, Cataluña destaca por ser la Comunidad autónoma que más formación en drogodependencias ofrece, seguida por la Comunidad de Madrid. Más de la mitad (el 53\%) de los centros universitarios españoles con titulaciones en medicina, enfermería, psicología y trabajo social no imparten docencia alguna en materia de drogas. Conclusiones: La formación pre-grado en drogodependencias sigue un proceso expansivo, aunque todavía está lejos de ser accesible para la totalidad de los alumnos de licenciaturas y diplomaturas con implicación profesional en el ámbito de las drogodependencias. Se requiere una mayor concienciación por parte de las autoridades académicas con vistas a ofrecer un mayor número de asignaturas relacionadas con las drogas en nuestras universidades y lograr una mayor interdisciplinariedad.

Palabras clave: Educación, Formación, drogodependencias, universidad.

\section{ABSTRACT}

Aim.- This paper presents an analysis and a description of pre-degree training in drug dependencies offered at Spanish universities. Method: The authors have reviewed the study plans of the 139 Spanish university faculties and colleges that offer degrees in medicine, psychology, nursing and/or social work. A total of 93 subjects exclusively on drugs, offered by 58 university faculties and colleges, have been included in this study. Results: Looking at the number of subjects available, we observe that the nursing degree is the one that offers the greatest number. Medicine and psychology degrees are in second and third place, with 26 and 24 subjects respectively. Social work lies in the last place with 6 subjects. Following a geographical breakdown, Catalonia is the Autonomic Community that provides the most training on drug-related issues, followed by the Community of Madrid. More than one half $(53 \%)$ of the Spanish university centres awarding degrees in medicine, nursing, psychology and social work do not offer any training on drug-related issues. Conclusions: Pre-graduate training in drug-related issues is following an expansionary process, although it is still far from being accessible to all university and college students professionally involved in drugrelated issues. A greater awareness by the academic authorities is required in order to be able to offer a higher number of drugrelated subjects in our universities and to leading to greater inter-disciplinarity.

Key words: Education, Training, Drug-addiction, University.

\section{INTRODUCCIÓNY JUSTIFICACIÓN}

I fenómeno de las drogodependencias constituye en la actualidad un problema de gran extensión y de una importante complejidad, repercutiendo a nivel económico, social, sanitario, jurídico, etc... Ante esta situación, es evidente la necesidad de implicar a diversos profesionales en el abordaje de los problemas asociados al consumo, es decir deben participar profesionales pertenecientes a la psicología, medicina 
y a la intervención social. Por lo tanto es importante no descuidar la formación de todos estos agentes involucrados en materia de drogodependencias. Esto nos lleva a preguntarnos ¿qué formación están recibiendo nuestros jóvenes universitarios?

A través de la enseñanza universitaria se pretende fomentar en los futuros profesionales la responsabilidad de contribuir con la sociedad buscando las soluciones más eficaces ante los múltiples problemas que afectan a la población, de ahí la importancia de comenzar la formación durante los estudios universitarios.

Desde organismos internacionales como la OMS (Comité de expertos de farmacodependencias. Serie Informes Técnicos, 551. Ginebra. 1974 y Nuevos métodos de educación sanitaria en la atención primaria de salud. Serie Informes Técnicos, 690. Ginebra.1983), también se ha hecho una mención especial a la necesidad de formar a personal capaz de desarrollar las funciones propias de las áreas de prevención, tratamiento y rehabilitación de los drogodependientes.

En España, el Ministerio de Sanidad y Consumo (1985) propone la formación en drogodependencias de los estudiantes universitarios, y en concreto de aquellas titulaciones implicadas profesionalmente de algún modo con la problemática de las drogas.

La escasez de publicaciones que abordan el tema de la formación pre-grado en materia de drogodependencias no nos permite conocer con exactitud el panorama anterior de la formación universitaria. Sin embargo, algunos estudios nos confirman el vacío formativo que ha existido en las universidades españolas en cuanto al tema de drogas se refiere. Del estudio de Ferrer y Sánchez de 1987, se desprende que la formación específica sobre este tema, en las facultades de Psicología españolas, no supera las 10 horas a lo largo de toda la carrera universitaria. Tres años más tarde se comprueba que en las titulaciones de Medicina y Enfermería de la Universidad de Alicante apenas se imparte formación en drogodependencias y en la Universidad Complutense de Madrid, se ofrecen entre 9 y 10 horas de formación en la carrera de Medicina y 12 horas en Enfermería (García Rodríguez, J.A. 1990).

En el estudio realizado por el profesor José Antonio García Rodríguez en 1988, en el que se planteaba conocer el nivel de formación de diferentes futuros profesionales, en concreto se centraba en las titulaciones de psicología, enfermería y medicina, y sus motivaciones e intereses en este tema, observamos que el 47,2\% considera escasa su formación/información y el 45,9\% la considera bastante, es más el 77,8\% afirma su voluntad de participar en cursos especializados sobre el tema de drogas. Uno de los datos más interesantes de este estudio es que el $62 \%$ de los universitarios cree necesario recibir formación relacionada con las drogodependencias. Consideramos este dato especialmente relevante si tenemos en cuenta que esta demanda surge desde los propios jóvenes.

Una de las mayores dificultades que aparecen a la hora de intentar tratar el problema de las drogodependencias es conseguir integrar a los diferentes profesionales que deben forman los equipos multidisciplinares necesarios para trabajar con mayor eficacia. Una cuestión que requiere de mayor atención es el abanico de profesionales que, de una forma u otra, están implicados en los diferentes ámbitos de las drogodependencias.

Son muchas las carreras profesionales relacionadas con el mundo de las adicciones, el presente estudio se centra en aquellas titulaciones de mayor relevancia en este tema, como son los estudios de Medicina, Psicología, Trabajo Social y Enfermería.

El objetivo principal que persigue este artículo consiste en describir el panorama formativo actual en drogodependencias en las universidades españolas. Este objetivo se desglosa en los siguientes objetivos específicos.

- Ofrecer información sobre el número de universidades españolas con formación en drogodependencias en las titulaciones de Medicina, Psicología, Enfermería y Trabajo Social, así como el porcentaje de éstas sobre el total de universidades que ofrecen dichas titulaciones.

- Conocer cuál es la media de créditos totales por asignatura de drogodependencias en cada una de las titulaciones.

- Profundizar en el tipo de asignaturas que abordan el tema de las drogas, es decir, especificar el porcentaje de asignaturas de libre elección, obligatorias, optativas y troncales relacionadas con este tema que aparecen en los planes de estudio.

- Revisar la formación universitaria en drogodependencias ofertada en España en las distintas Comunidades Autónomas.

\section{MATERIAL Y MÉTODO}

\section{Muestra}

La muestra analizada en la presente investigación se compone de los planes de estudio de las titulaciones de medicina, psicología, enfermería y trabajo social impartidas en la universidades españolas. Siguiendo el procedimiento que se describe a continuación, fueron identificadas un total de 139 facultades y escuelas universitarias. 
En primer lugar se identificaron aquellas titulaciones que presentaban una mayor carga docente en materia de drogodependencias. Siguiendo un criterio de presencia profesional en el ejercicio de la reducción de la oferta y la demanda de drogas, inicialmente se seleccionaron un total de 6 titulaciones, a saber: medicina, psicología, enfermería, trabajo social, derecho y farmacia. Tras comprobar en cada una de ellas la presencia de docencia en materia de drogodependencias, fueron descartadas las titulaciones de derecho y de farmacia por no ofrecer asignaturas de drogodependencias entre sus planes de estudio.

Por lo tanto, la muestra de trabajo quedó constituida por 93 asignaturas, distribuidas entre las cuatro titulaciones incluidas y coincide con el universo de la población objeto de estudio, es decir la totalidad de la docencia pre-grado en dichas titulaciones.

\section{Universidades}

Para proceder a su identificación se recurrió al listado publicado en la página web del Ministerio de Educación en el apartado de la Secretaria General del Consejo de Coordinación Universitaria (http://www. mec.es/consejou/oferta/index.html) en la que se incluyen un total de 76 universidades distribuidas de la siguiente forma:

\begin{tabular}{lc}
\hline Universidades públicas & 50 \\
\hline Universidades privadas y de la Iglesia Católica & 22 \\
\hline Universidades No presenciales & 2 \\
\hline Universidades internacionales & 2 \\
\hline
\end{tabular}

\section{Titulaciones}

La página de la Secretaria General del Consejo de Coordinación Universitaria, anteriormente referenciada, (http://www.mec.es/consejou/oferta/index.html) ofrece la posibilidad de una búsqueda por titulaciones. De esta forma, fueron identificados los centros universitarios que imparten las titulaciones de medicina, enfermería, psicología y trabajo social.

\section{Variables estudiadas}

La recogida de información se realizó a través de dos plantillas que fueron cumplimentadas para cada plan de estudios y para cada universidad, respectivamente. Los datos recogidos se volcaron en una base de datos.
La información recogida en referencia a los planes de estudio fue: 1) Nombre de la asignatura; 2) Tipo de asignatura (troncal, obligatoria, optativa o libre configuración), 3) Créditos totales (prácticos y teóricos). Conviene aclarar en este punto, que según el sistema español de ordenación académica regulado por el Real Decreto 1497/87 del 27 de Noviembre, en su artículo 2 pto. 7, un crédito "es la unidad de valoración de las enseñanzas, que corresponderá a diez horas de enseñanza teórica, práctica o de sus equivalencias. La obtención de los créditos estará condicionada a los sistemas de verificación de los conocimientos que establezcan las universidades." 4) Curso; 5) titulación; 6) Departamento responsable; 7) Universidad; 8) Descripción; 9) Programa.

En cuanto a los centros universitarios se compiló la siguiente información: 1) Nombre del centro; 2) Titulaciones impartidas (psicología, trabajo social, medicina y/o enfermería) indicando cuál de ellas oferta docencia en drogodependencias.

\section{Método}

El acceso a la información se hizo a través de las páginas web de las 58 universidades incluidas en el estudio, revisando cada una de las variables expuestas en el apartado anterior. Se recurrió al contacto telefónico o al correo electrónico en aquellos casos en los que la información ofrecida por las páginas web era insuficiente o confusa.

\section{RESULTADOS}

A continuación pasamos a describir los resultados obtenidos en el presente estudio, los cuales vamos a dividir en los siguientes apartados: 1) Tipo de asignatura, 2) Número de créditos, 3) Resultados por titulaciones y 4) Resultados por comunidades autónomas.

\section{Tipo de Asignatura}

Con respecto al tipo de asignatura, se puede apreciar en el gráfico 1 que la gran mayoría de asignaturas sobre drogas y drogodependencias recae en las optativas. En concreto el $75,27 \%$ de las asignaturas con contenidos monográficos sobre drogas ofertadas en las universidades españolas con formación en enfermería, psicología, medicina y trabajo social, son asignaturas optativas. Entendidas como aquellas libremente establecidas por cada universidad, que se incluyen en el correspondiente plan de estudios para 
que el alumno escoja entre las mismas. Normalmente las asignaturas optativas suelen agruparse para formar itinerarios / especialidades. El 17,20\% del total de asignaturas dedicadas a las drogodependencias correspondería a créditos de libre elección, éstos pueden ser asignaturas de otras titulaciones u optativas de la propia titulación. También se consideran créditos de libre elección aquellos que se pueden obtener a través de cursos, congresos, seminarios, etc.. reconocidos por el Consejo de Gobierno de la Universidad correspondiente, pero éstos no han sido contemplados en nuestra revisión. Se observa un pequeño porcentaje de asignaturas obligatorias, 7,52\%. Las denominadas asignaturas obligatorias, las establece cada universidad de modo que no necesariamente figuran en los planes de estudio conducentes al mismo título en otra universidad. En casi todas las titulaciones revisadas se oferta alguna asignatura obligatoria relacionada con las drogodependencias, excepto en psicología.

Por último, destacar que no se han encontrado asignaturas de carácter troncal, dedicadas a las drogodependencias. Estas asignaturas son de obligatoria inclusión en todos los planes de estudio que conduzcan a un mismo título oficial en cualquier universidad.

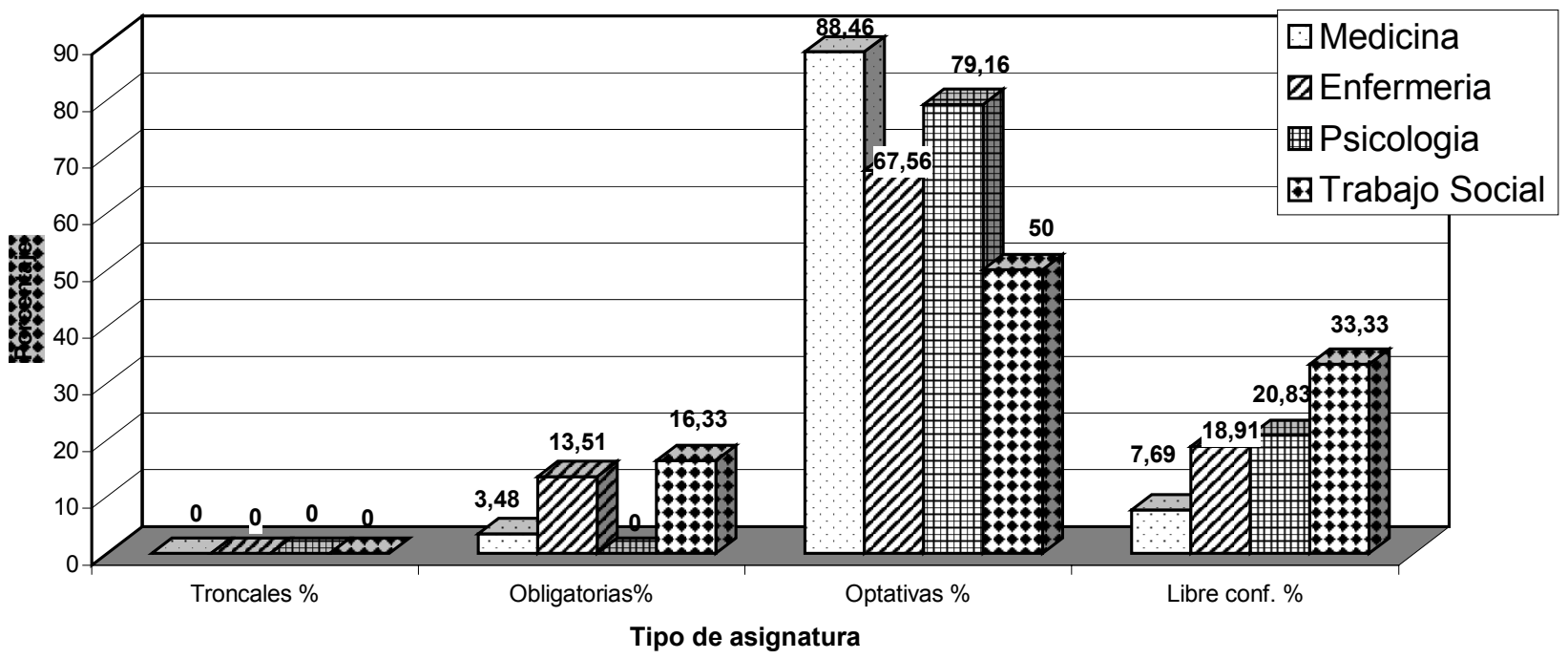

Gráfico 1

Asignaturas con contenidos monográficos sobre drogas según titulación y tipo de asignatura

\section{Número de créditos}

Si analizamos el número de créditos correspondiente a las asignaturas de drogodependencias que se imparten en las distintas titulaciones, observamos que la media total de créditos por cada asignatura de drogodependencias es de 4,5 créditos. La titulación que oferta la mayor media de créditos por asignatura con contenidos relacionados con las drogas es psicología, cuya media es de 4,9 créditos, seguida muy de cerca por la carrera de enfermería con una media de créditos totales de 4,56. En trabajo social se imparte una media de 4,37 créditos y en último lugar se encuentra medicina que imparte una media de 4,17 (véase gráfico 2).

En general podemos afirmar que no existen grandes diferencias entre las cuatro titulaciones en cuanto al número de créditos de las asignaturas de drogodependencias se refiere.
En el apartado anterior hemos comentado que el porcentaje mayor de asignaturas que abordan el tema de las adicciones son optativas, por lo tanto la mayor parte de estos créditos constituyen créditos optativos dentro del plan de estudios de las titulaciones tratadas en nuestro estudio.

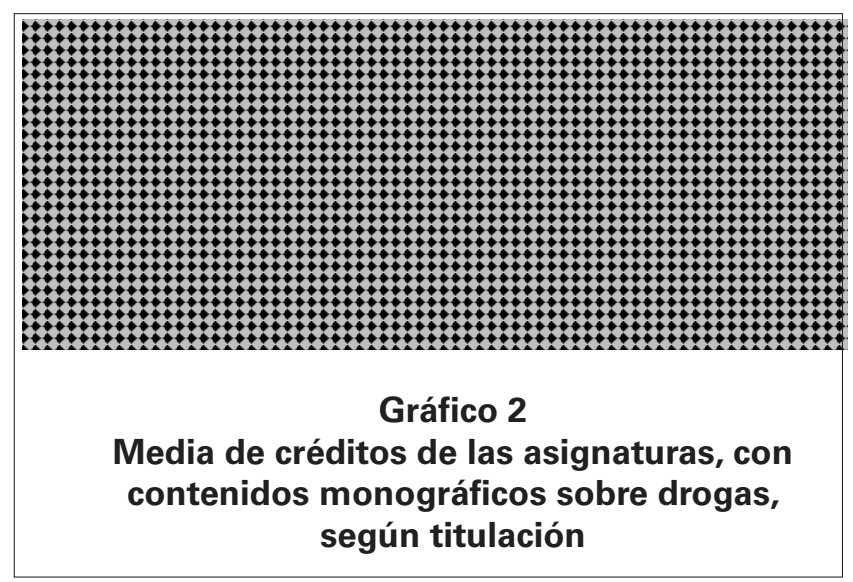




\section{3.- Resultados por titulaciones.}

Atendiendo a cada una de las titulaciones los resultados obtenidos han sido los siguientes:

\section{Titulación de Medicina}

En la titulación de medicina observamos que de un total de $\mathbf{2 7}$ universidades españolas que cursan esta carrera, 20 incluyen asignaturas monográficas sobre drogodependencias en sus planes de estudio, equivalente a un porcentaje del $74 \%$ de las universidades. Esto indica que en un porcentaje muy alto de las universidades que imparten la titulación de medicina se ofrece formación en drogodependencias.

A través de estas 20 universidades que incluyen formación en drogas se imparte un total de 26 asignaturas que abordan el tema de las conductas adictivas de forma monográfica, con una media de créditos totales por cada una de estas asignaturas de 4,17 créditos. Si observamos el gráfico 3, encontramos que 23 de estas asignaturas son optativas (un $88,46 \%$ ), una es obligatoria $(3,85 \%)$, dos son de libre configuración $(7,69 \%)$ y no poseen ninguna asignatura de drogodependencias que sea troncal. Como podemos observar la inmensa mayoría de estas asignaturas son optativas.

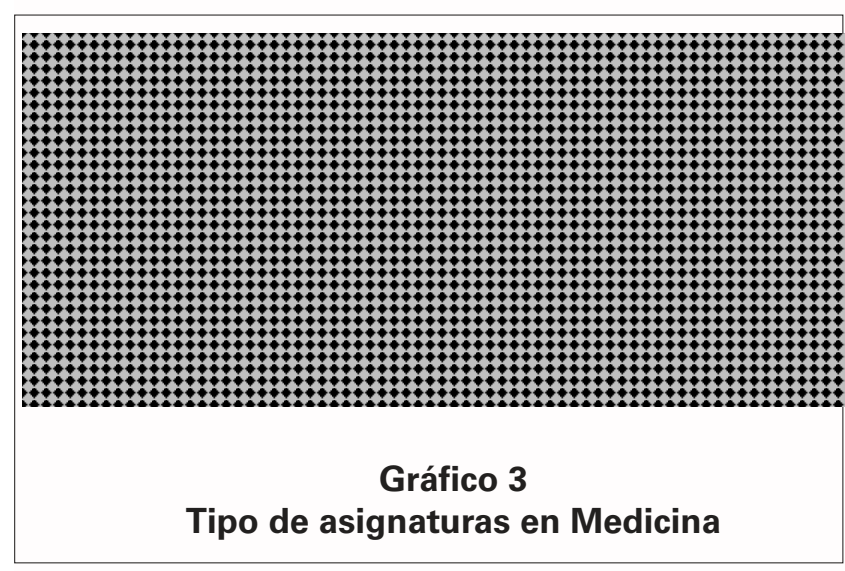

La formación universitaria en drogodependencias a través de la titulación de medicina se ofrece en las comunidades autónomas de Andalucía, Canarias, Cantabria, Castilla-La Mancha, Castilla y León, Cataluña, Comunidad Valenciana, Extremadura, Galicia, Madrid, Murcia, y País Vasco.

\section{Titulación de Enfermería}

La titulación de enfermería se imparte en 49 universidades españolas, de las cuales 21 ofrecen formación en drogodependencias, siendo el porcentaje de universidades con formación del $42 \%$.
Encontramos un total de 37 asignaturas dedicadas exclusivamente a las drogodependencias cuya media de créditos totales por cada una de ellas es de 4,56 créditos. De nuevo la mayor parte de estas asignaturas son optativas, 25 de ellas $(67,56 \%)$, como se puede apreciar en el gráfico 4.7 asignaturas son de libre configuración $(18,91 \%)$ y 5 son obligatorias $(13,51 \%)$.

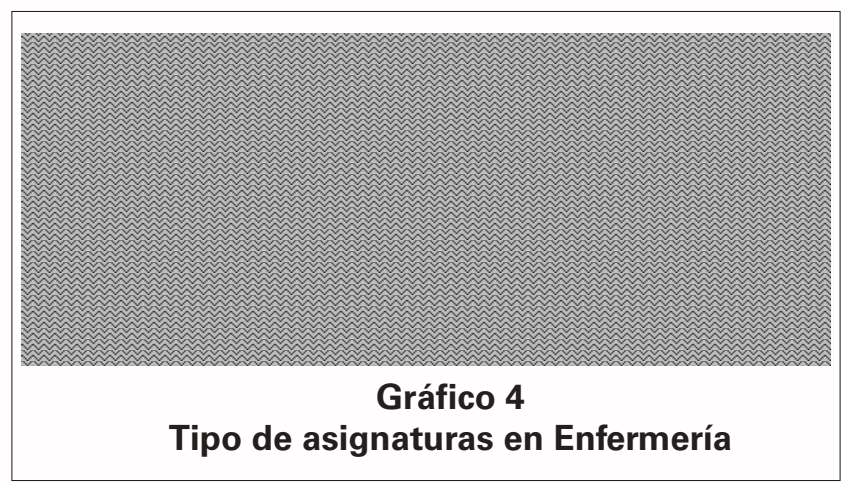

Encontramos formación universitaria en drogodependencias a través de la titulación de enfermería en las siguientes comunidades autónomas: Andalucía, Asturias, Baleares, Canarias, Castilla La- Mancha, Castilla y León, Cataluña, Comunidad Valenciana, Galicia, La Rioja, Madrid y Murcia.

\section{Titulación de Psicología}

En la titulación de psicología observamos un porcentaje del $53 \%$ de universidades españolas con formación en drogodependencias, que corresponde a 16 universidades que imparten asignaturas que abordan el tema de las drogas y drogodependencias de un total de 32 universidades que cursan esta carrera. Es decir, en más de la mitad de universidades que incluyen los estudios de psicología se ofrece esta formación específica.

Encontramos un total de 24 asignaturas de drogodependencias con una media de 4,9 créditos. El mayor porcentaje lo siguen ocupando las asignaturas optativas, 19 de ellas $(79,16 \%)$, mientras que 5 son asignaturas de libre configuración $(20,83 \%$ ) y no encontramos ninguna asignatura de carácter troncal u obligatorio relacionada con las drogodependencias (véase gráfico 5).

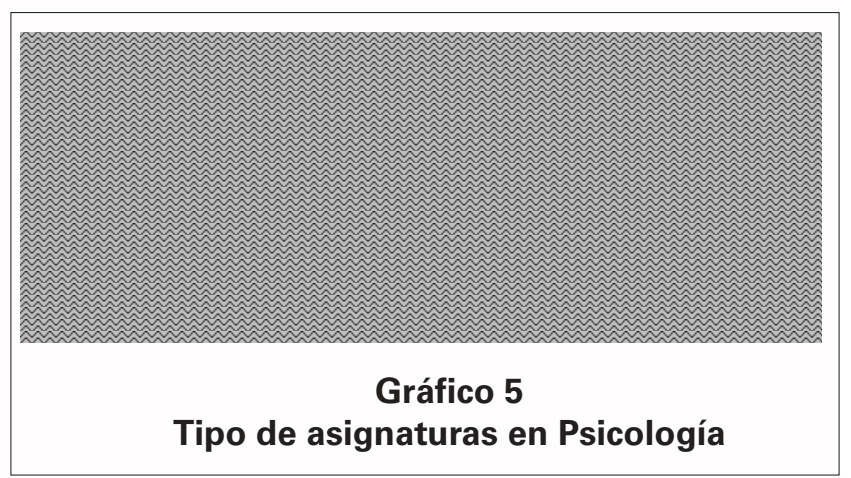


Las comunidades que ofrecen formación en drogodependencias mediante la titulación universitaria de psicología son: Andalucía, Asturias, Baleares, Castilla y León, Cataluña, Comunidad Valenciana, Galicia, Madrid y País Vasco.

\section{Titulación de Trabajo Social}

Por último, la Diplomatura de trabajo social se encuentra en 31 universidades españolas, de las cuales tan solo 6 ofrecen formación en drogodependencias, siendo el porcentaje de universidades con formación el 19,35\%.

Este limitado número de universidades ofrece un total de 6 asignaturas que tratan las drogas, con una media de 4,37 créditos totales por cada asignatura.

De estas seis asignaturas, 3 de ellas son optativas, es decir el 50\%, 2 son de libre configuración (33,33\%)

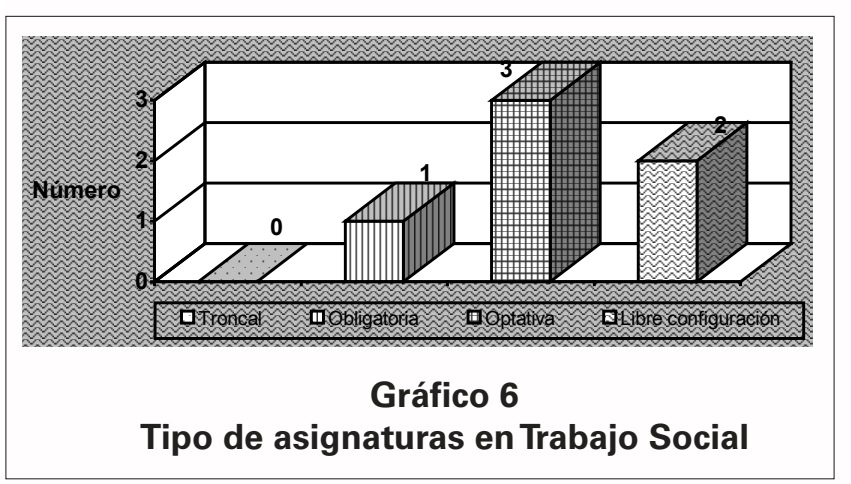

y una es de carácter obligatorio (16,66\%). Esto se puede observar en el gráfico 6.

Muy pocas comunidades autónomas ofrecen formación universitaria en drogas a través de la titulación de trabajo social, encontramos este tipo de formación en las comunidades de: Asturias, Baleares, Canarias, Comunidad Valenciana y Murcia.

Los gráficos 7 y 8 nos muestran que la titulación que ofrece una mayor formación universitaria en drogodependencias es enfermería, con 37 asignaturas ofertadas en 21 universidades que constituyen el $42 \%$ de las universidades españolas donde se puede cursar esta titulación. Además su media de créditos totales por cada asignatura es la segunda más alta (4,56 créditos por asignatura).

En segundo lugar se sitúa medicina, con un total de 26 asignaturas impartidas en 20 universidades que representan el $74 \%$ de la totalidad de centros universitarios que ofrecen esta titulación. Su media de créditos es de 4,17 créditos por asignatura.

Los alumnos de psicología cuentan con 24 asignaturas repartidas en 16 universidades, que representan el $53 \%$ de los centros universitarios. La media de la carga docente es de 4,9 créditos por asignatura, la más elevada de las cuatro titulaciones.

Por último, la diplomatura de trabajo social ofrece 6 asignaturas en 6 centros, lo cual supone el 19,35\% de los centros universitarios españoles. El número de créditos por asignatura se sitúa en 4,37 de media.

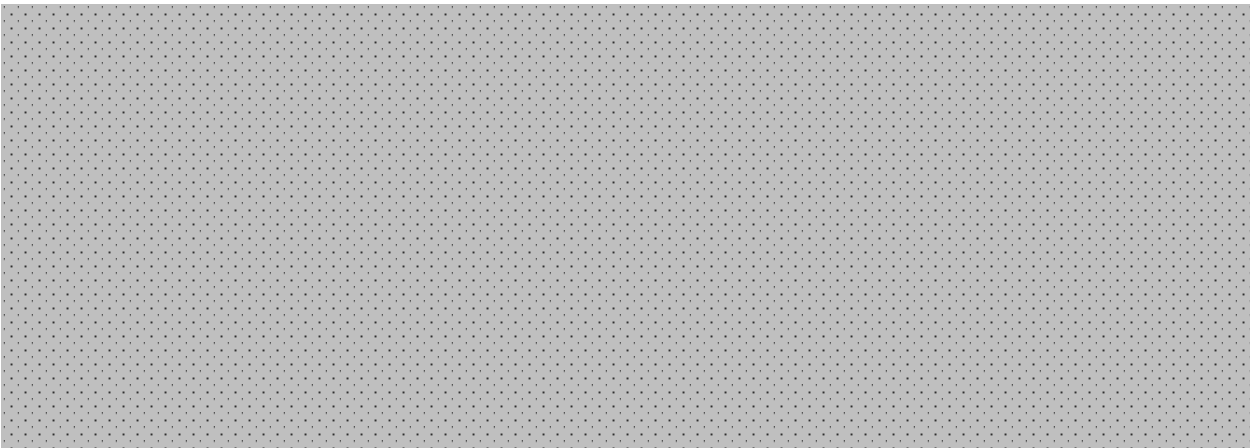

Gráfico 7. Porcentaje de universidades con formación en drogodependencias en cada titulación y número sobre el total de centros que ofertan la titulación.

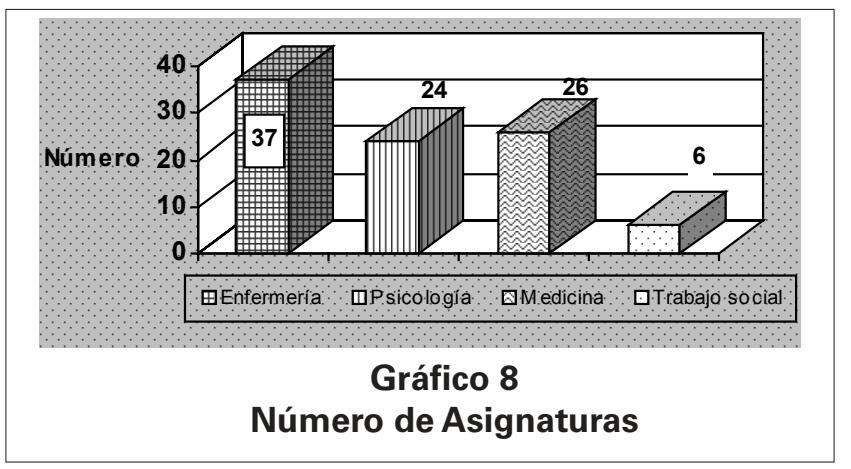

\section{Resultados por Comunidades Autónomas.}

En cuanto a las comunidades autónomas, observamos que las titulaciones elegidas para el presente trabajo se distribuyen en las comunidades de Andalucía, Asturias, Baleares, Canarias, Cantabria, Castilla la Mancha, Castilla León, Cataluña, Comunidad Valenciana, Extremadura, Galicia, La Rioja, Madrid, Murcia y País Vasco. No habiéndose encontrado formación en los centros universitarios de Aragón y Navarra. Aten- 
diendo al número de asignaturas, Cataluña destaca por ser la comunidad autónoma que más formación en drogodependencias oferta, con un total de 17 asignaturas, en las titulaciones de medicina, enfermería y psicología, que representan el $20 \%$ del total de asignaturas ofertadas por todas las universidades españolas. A continuación sigue la comunidad de Madrid, que constituye el $14 \%$ de la formación en drogodependencias, a través de esas mismas titulaciones. En la interpretación de los datos hemos de tener en cuenta que las comunidades de Cantabria y Extremadura únicamente ofertan formación en drogas en la titulación de medicina y La Rioja, en enfermería, por lo tanto sus porcentajes son del $2 \%, 1 \%$ y $1 \%$, respectivamente, siendo las comunidades que menos formación en materia de drogodependencias imparten. El gráfico 9 muestra la distribución porcentual de las asignaturas en las cuatro titulaciones por comunidades autónomas.

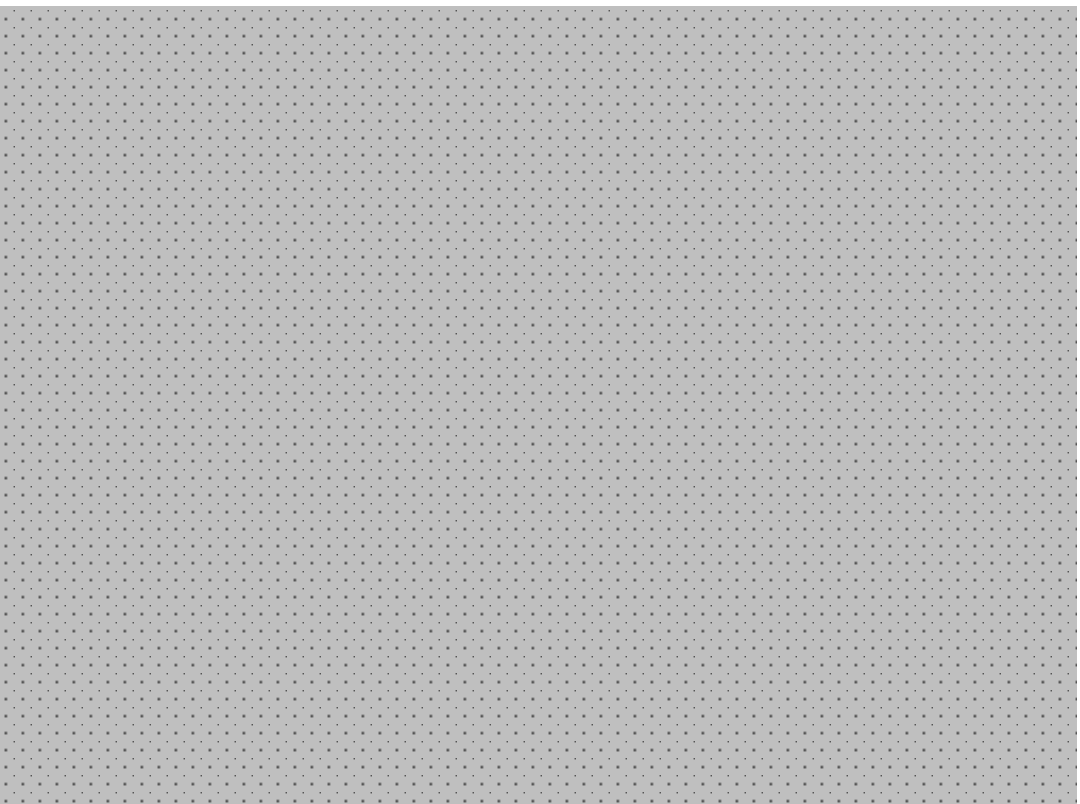

Gráfico 9

Distribución porcentual del número de asignaturas ofertadas por Comunidades Autónomas.

\section{DISCUSIÓNY CONCLUSIONES}

Es un hecho que en los últimos años se han producido importantes cambios en cuanto a la formación en drogodependencias se refiere. Una muestra de ello, la encontramos al comparar nuestros resultados con los datos obtenidos en 1987 por Ferrer y Sánchez. En su estudio sobre la formación en drogodependencias, observamos que dieciséis años después la media de horas de formación a lo largo de toda la carrera universitaria de psicología, ha aumentado de 10 horas a 49 horas totales de formación. Como afirma el Ministerio de Sanidad y Consumo (1985, p.33) se hace necesario "asegurar la correcta formación en drogodependencias de los estudiantes universitarios de profesiones relacionadas con este tema (especialmente sanitarios, sociólogos, psicólogos, educadores, trabajadores sociales y profesionales del derecho)".
Este avance con respecto a la formación pre-grado en materia de drogas, es realmente limitado si tenemos en cuenta el aumento de la demanda laboral de profesionales especializados en el campo de las drogodependencias que venimos observando en los últimos años. La prevención, tratamiento y reinserción del drogodependiente continúa profesionalizándose y regularizándose mediante la creación y el fortalecimiento de nuevas estructuras y dispositivos de atención y de un cuerpo normativo que vela por la calidad de los servicios de prevención y atención al usuario de drogas. La preocupación de la sociedad y de la administración pública por atender este fenómeno ha impulsado la creación de nuevos puestos de trabajo y la estabilidad de los ya existentes. Atendiendo a este panorama socio-laboral consolidado, entendemos que es obligación de la universidad responder a la creciente demanda laboral requerida por la sociedad, ofreciendo la oportunidad a sus estudiantes de familiarizarse con aquellas áreas de conocimiento que presentan importantes implicaciones profesionales. 
Especial mención merece el "tipo de asignatura", pues aproximadamente el $91 \%$ de la formación pregrado española en drogodependencias, depende de la propia elección del alumno. Es decir, hoy en día la formación se limita, prácticamente de forma exclusiva, a asignaturas optativas y de libre elección, de forma que desde las universidades no se asegura que los estudiantes adquirirán, al menos, las nociones básicas capaces de ofrecer los conocimientos suficientes. Conocimientos que les permitirían por una parte, elegir su perfil profesional y por otra, cubrir las futuras demandas laborales relacionadas con las drogodependencias.

Si bien es cierto que la intervención en el ámbito de drogas se plantea desde un modelo bio-psicosocial, la realidad nos dice que no hemos logrado conseguir equilibrar la participación de los tres componentes del modelo. Es decir, tanto en la práctica profesional como en la formación universitaria, observamos un mayor peso en lo "biológico". Un reflejo de esta desigualdad del modelo bio-psico-social en la práctica profesional, lo encontramos en los grupos de profesionales que forman los equipos asistenciales, donde se pondera la presencia de profesionales de la parte biológica, como son el médico y el enfermero y de la parte psicológica con el psicólogo, frente al descuido del área social con la escasa presencia de la figura del trabajador social. Si nos remitimos a los datos de nuestra investigación, comprobamos que los licenciados en medicina, seguidos de los diplomados en enfermería, son los profesionales que más formación reciben en materia de drogas, sin embargo, los diplomados en trabajo social son a los que menos formación en drogodependencias se les ofrece desde las universidades españolas.

Los resultados de la investigación ponen de manifiesto que más de la mitad (el 53\%) de los centros universitarios españoles con titulaciones en medicina, enfermería, psicología y trabajo social no ofrecen docencia alguna en materia de drogas. Según el Informe $N^{\circ} 5$ del Observatorio Español sobre Drogas en el año 2000 se produjeron 49.000 admisiones a tratamiento. Este dato nos hace reflexionar sobre el hecho de que la alta prevalencia de la patología bio-psicosocial producida por el consumo de drogas, parece no suponer motivo suficiente para que se incluyan en todos los planes de estudio asignaturas relacionadas con las drogodependencias.

Por último, podemos concluir que, atendiendo a los resultados de la presente investigación, consideramos que sería realmente necesario que las instituciones académicas incrementaran sus esfuerzos con vistas a conseguir estos importantes objetivos: ofrecer un mayor número de asignaturas relacionadas con las drogas en nuestras universidades españolas y lograr una mayor interdisciplinariedad.

\section{REFERENCIAS}

Aizpiri, J. (1984) La formación de los profesionales en el campo de las drogodependencias. DAK, Gobierno Vasco. $52 \mathrm{pp}$.

Álvarez F.J. (2001). La formación continuada en Drogodependencias y las Revistas especializadas en Drogodependencias. Revista Española de Drogodependencias. 26 (2), 135-145.

Becoña, E., Llopis, J. y Borbes J. (2000). La formación continuada en drogodependencias. Adicciones. 12 (1), 3-5.

Bordons, E. (1988). La universidad y la formación de profesionales en drogodependencias. Comunicación a las II Jornadas Estatales de Psicología, Drogodependencias y Sociedad. Granada.

Bordons, E. (1989). La división de formación e investigación de la F.A.D. Ponencia presentada a las I Jornadas sobre actuación de las Instituciones en materia de drogodependencias. Córdoba.

Cano, L. (1993). La formación de los profesionales en España en materia de drogodependencias: evolución, balance y perspectivas. En J.A. García y J. Ruíz. Tratado sobre prevención de las drogodependencias. Edex. Koletoboa. FAD. Gobierno Vasco.

Escames, J. (1994). La formación universitaria en drogodependencias. Revista Española de Drogodependencias. 19 (3), 187-190.

Ferrer, X. (1981). La educación sobre las drogas en la Universidad y Escuelas Especiales para los profesionales implicados. Drogalcohol. VI. (2). 117-125.

Ferrer, X. y Sánchez, M. (1987). La Formación de profesionales en materia de drogodependencias. Comunidad $y$ Drogas. 5/6, 63-82.

García-Rodríguez, J. A. (1988). Proyecto de formación de profesionales en drogodependencias: Informe de Necesidades. Instituto Complutense de Drogodependencias (inédito). Madrid.

García-Rodríguez, J. A. (1989a). Formación pregrado de drogodependencias. Ponencia presentada a las I Jornadas sobre actuación de las Instituciones en materia de drogodependencias. Córdoba.

García-Rodríguez, J. A. (1989b). Prevención de las drogodependencias: prevención específica versus inespecífica. Revista Adicciones, vol. 1,2, 121-129.

García-Rodríguez, J. A. (1990). Formación en drogodependencias. Revista Adicciones, vol. 2, 4, 291-302.

Guillem, J. L.; Cuquerella, M.A.; Hernández, M.; Perez, J.F.; Raya, N.; Conesa, LI.; Vila, M.L. (1997). Formación en adicciones en el programa MIR (Médico Interno Residente) de psiquiatría en España. Resultados de una encuesta. Adicciones. 9 (4), 571-578.

Lacoste.J.A. (1988). La Universidad ante un reto profesional: las drogodependencias. XVI Jornadas de Socidrogalcohol. Valladolid.

Mayor, L. y Cano, L. (1992). Análisis y perspectivas de la formación de profesionales en drogodependencias. Revista Española de Drogodependencias.17 (3), 155-162. 
Mendoza, R. (1985). Toxicología de la drogadicción. Díaz de Santos. Madrid.

Ministerio de Sanidad y Consumo (1985) Delegación del Gobierno para el Plan Nacional sobre drogas. Madrid.

Observatorio Español sobre Drogas (2001) Informe $N^{\circ} 4$ Delegación del Gobierno para el Plan Nacional sobre Drogas. Madrid.

Observatorio Español sobre Drogas (2002) Informe No 5 Delegación del Gobierno para el Plan Nacional sobre Drogas. Madrid.

O.M.S. (1974). Comité de expertos de farmacodependencias. Serie Informes Técnicos, 551. Ginebra.

O.M.S. (1683). Nuevos métodos de educación sanitaria en la atención primaria se salud. Serie Informes Técnicos, 690. Ginebra.
Sánchez Turet, M. (1999). Formación en drogodependencias en las universidades españolas. Revista Española Drogodependencias. 24 (1), 1-2.

Sierra, E. (1983). Formación de profesionales en el campo de las drogodependencias. I Jornadas Municipales de Prevención de las drogodependencias. Mimeo, Valencia.

Vega, A. (1986). El psicólogo ante un reto social: las drogodependencias. Papeles del Psicólogo. Revista del Colegio Oficial de Psicólogos. 4 (24) 12-14.

Vega, A. (1999). ¿Dónde queda la formación de los profesionales ante la cuestión de las drogas? Revista Española de Drogodependencias. 24 (4). 332-335. 
